\title{
MIGRACIÓN Y DERECHOS HUMANOS. EL CASO DE LOS MIXTECOS*
}

\author{
Por \\ María Eugenia Anguiano**
}

\begin{abstract}
RESUMEN
En este ensayo se pone de manifiesto la situación de los derechos humanos de los migrantes, en particular la de los indígenas mixtecos, puesto que éstos constituyen el grupo más numeroso de migrantes con experiencia de trabajo en el noroeste de México y la costa oeste de Estados Unidos.

Se reseñan, además, las condiciones generales que en las comunidades de origen han obligado a los indígenas de la Mixteca oaxaqueña a emigrar hacia el norte del país.

La inserción de los mixtecos en el mercado laboral ya no se limita a los empleos tradicionales, sino que se ha ampliado a otros sectores de la economía formal e informal en las ciudades del noroeste y Pacífico norte.

Por último se destaca la presencia del indígena mixteco en la reproducción de su cultura y su integración en organizaciones sindicales.
\end{abstract}

\begin{abstract}
This essay exposes the situation of the migrant's human rights, in particular those of the Mixtecs indigenous, since they integrate the largest group of indians migrants with work experience in the north west of Mexico and the cast coast of the United Siates.

Are also treated the general conditions for which the Mixtecs are forced to emigrate from their native communities up to the north of Mexico.

The integration of the Mixtecs into the labor market is not limited to the traditional line of employment anymore, but it has developed toother segments of the formal and informal economy of cities in the northwest and throughout the Pacific coast.

It is also outlined the presence of the Mixtecs in the reproduction of their culture and their integration to the sindicalized organizations.
\end{abstract}

\section{INTRODUCCIÓN}

En los análisis sobre el fenómeno de la migración se ha hecho énfasis en los factores socioeconómicos que determinan los movimientos territoriales de la población; se han investigado las motivaciones individuales y familiares que inciden en la selectividad entre quienes migran y quienes

- Agradezco la colaboración de Alberto Hemández en la elaboración de este trabajo.

** Investigadora del Colegio de la Frontera None. 
no; se han analizado los cambios que han experimentado las corrientes migratorias, interna e internacional, en su dinámica y composición interna en distintas épocas. En ese amplio panorama de investigación, un aspecto recientemente discutido es la situación de los derechos humanos de los migrantes. En este ensayo interesa en particular la de aquéllos que se trasladan desde la Mixteca oaxaqueña "hacia el norte", que comprende el centro occidente y noroeste del país y la costa oeste de los Estados Unidos.

Aunque los movimientos migratorios han sido una constante en la vida económica y social de la población de las comunidades rurales del estado de Oaxaca, en la década de los ochenta, los indígenas mixtecos representaron uno de los grupos de trabajadores étnicos más numerosos que se desplazaron al noroeste del país, y en años recientes hacia los estados de California, Oregon y Washington, en los Estados Unidos. Este movimiento poblacional se ha desarrollado paralelo a la conformación de nuevas unidades productivas agrícolas de carácter empresarial y al incremento de cultivos de exportación en el noroeste de México, procesos que han determinado cambios en el destino de esta corriente migratoria y en su composición interna. ${ }^{1}$ Respecto al destino laboral a fines de los setenta, una parte de los jomaleros agrícolas del noroeste empezaron a dirigirse a nuevos mercados de trabajo y a emplearse en cultivos que demandaban su ocupación intensiva y estacional: a desplazarse de Nayarit, Sinaloa y Sonora hacia al valle de San Quintín, Baja California, y también a los campos agrícolas del estado de California y a las áreas frutícolas de los estados de Oregon y Washington, de cultivos como el algodón a las hortalizas y frutas frescas. Su composición interna también se transformó: cada vez más se integran al duro trabajo en los campos hombres, mujeres y niños procedentes de comunidades indígenas del estado de Oaxaca, proceso que ha determinado el movimiento de familias e incluso comunidades enteras.

La migración de este grupo étnico, en tanto desplazamiento laboral, tiene dos características: a) es un fenómeno de carácter masivo, por la cantidad de personas y el número de salidas y retornos que efectúan; y b) el traslado entre puntos geográficamente tan distantes no constituye una elección entre otras alternativas de ocupación, sino una necesidad y, en ocasiones, la última o única opción que tienen para sobrevivir. La creciente

1 Como Rodolfo Corona señala "Los movimientos territoriales de población constituyen un fenómeno dinámico y complejo, del todo ligado a la evolución socioeconómica de un país o región. En lo fundamental, las migraciones se originan en factores económicos, pues generalmente los individuos se desplazan al reconocer la dificultad para solucionar sus carencias o mejorar sus condiciones materiales de vida en sus lugares donde habitan, junto con la identificación de posibilidades para satisfacer sus privaciones o alcanzar sus expectativas económicas en otros sitios." (Ver Corona, 1988: 7). 
emigración de mixtecos, a miles de kilómetros de sus comunidades, refleja la pobreza en que viven en sus lugares de origen, agravada por los costos económicos y sociales de la migración que deben cubrir el trabajador, su familia y su comunidad.

En el presente ensayo, los jornaleros indígenas mixtecos son considerados trabajadores migratorios cuyo carácter étnico imprime características peculiares al proceso de su integración social, que los hacen más vulnerables en la defensa de sus derechos civiles, laborales y sociales que a otros grupos de trabajadores migrantes, tanto en México como en Estados Unidos.

En las dos primeras secciones del texto, se reseñan las condiciones generales que en las comunidades de origen han obligado a los indígenas de la Mixteca oaxaqueña a emigrar hacia el norte del país, así como su ubicación selectiva en los mercados laborales; selectividad que ha sido ampliamente vinculada con las estrategias de reproducción social de este grupo. Las dos secciones posteriores están dedicadas a presentar algunas de las formas más frecuentes de violación a sus derechos humanos y las limitaciones que presentan las acciones gubernamentales emprendidas para combatirlas; esta situación ha servido de estímulo para la propia organización de los mixtecos en defensa de sus derechos como trabajadores, como migrantes y como grupo étnico.

\section{DE LA MIXTECA A LA FRONTERA NORTE}

La Mixteca está ubicada en la región suroeste del Pacífico; abarca principalmente el occidente de Oaxaca y se extiende a los estados de Guerrero y Puebla (Butterworth, 1975: 21- 23). La Mixteca oaxaqueña ha sido considerada como una de las regiones más pobres de México; con nueve meses de sequías y tres de lluvias inciertas y mal temporal, sólo el $7.2 \%$ de su superficie es agrícola y menos del uno por ciento, de riego. De acuerdo con información del programa Lluvia, tequio y alimentos (Gobierno del estado de Oaxaca, 1987), en menos de 30 años la Mixteca se ha convertido en una estepa árida, en donde subsisten apenas unos cuantos pequeños pueblos en los pocos valles irrigados. El creciente deterioro agrícola, resultado de un proceso gradual de erosión y disminución progresiva de las tierras de cultivo, ha determinado altas tasas de emigración de su población, constituyéndose en la principal zona de expulsión del estado de Oaxaca. La combinación de la pobreza del suelo y el rápido crecimiento de la población han acelerado el éxodo rural.

Los niveles de vida de los campesinos mixtecos son precarios. La región ocupa los primeros lugares en mortalidad infantil y analfabetismo, 
con bajos niveles de inversión por habitante. Se encuentra desprovista de caminos, escuelas y aulas (informe de la Comisión de Asuntos Indígenas, 1983). Sus ingresos reales fundamentalmente provienen de la migración: el $80 \%$ del ingreso mixteco es generado fuera de esta región. ${ }^{2}$

Las explotaciones menores de dos hectáreas de tierra empobrecida y erosionada con que cuentan la mayoría de las familias campesinas de la Mixteca, no les proporcionan recursos para vivir ni aun al nivel mínimo de subsistencia. En estas condiciones, los mixtecos se han visto obligados a salir de sus comunidades de origen y convertirse en trabajadores asalariados en el campo y en las ciudades. El mercado de trabajo formal e informal se convierte en la única alternativa para la reproducción individual y familiar. En el caso de los trabajadores agrícolas, su precaria subsistencia depende de empleos transitorios y eventuales determinados por los ciclos productivos, que los obligan a permanecer largas temporadas fuera de sus comunidades o a seguir las rutas que marcan los cultivos en el noroeste de México y en la costa Pacífico de los Estados Unidos.

La emigración de mixtecos desde sus comunidades hacia los mercados laborales rurales y urbanos del interior del país no constituye un fenómeno novedoso. Lo que destaca es el carácter internacional que ha adquirido su movimiento: en la actualidad los mixtecos constituyen el grupo más numeroso de migrantes indígenas con experiencia de trabajo en el noroeste de México y la costa oeste de Estados Unidos. Se estima que de cerca de 100 mil jornaleros agrícolas oaxaqueños que circulan en los estados de Sinaloa, Sonora, Baja California y Baja California Sur aproximadamente el $80 \%$ son mixtecos. ${ }^{3}$ En los estados de California, Oregon y Washington su número se calcula en más de 20 mil (Zavin, et al., 1990). En estas regiones, los jornaleros son contratados en forma cíclica para actividades de corte, cultivo y cosecha de productos como jitomate, cítricos, hortalizas, frutas frescas, vid y algodón, entre otros (ver cuadro 1).

En su largo recorrido en busca de oportunidades laborales, la necesidad de los mixtecos de desplazarse a largas distancias y el progresivo desarraigo de sus tierras, los han llevado a establecer asentamientos permanentes

2 En el año de 1980 el Consejo Estatal de Población estimó que de cada diez mixtecos, tres emigraban en forma definitiva, cuatro temporalmente y solamente tres permanecían en la región. De los migrantes, 39\% se dirigían al Distrito Federal, 22\% al Estado de México y $17 \%$ a Veracruz. El restante $22 \%$ emigró a otros estados del país y al extranjero (Besserer, 1989: 16-18).

En el valle de San Quintín, en Baja Califomia, cerca del 80\% de los jomaleros agrícolas son mixtecos. En los campos agrícolas del valle de Culiacán, en Sinaloa, laboraron más de 83 mil trabajadores en la temporada 1988-1989. De ellos, más del 95\% eran trabajadores eventuales y el $80 \%$ migrantes; la tercera parte de este flujo estaba constituido por jornaleros mixtecos (véanse: Anguiano, 1986: 49-52; Gobierno del estado de Sinaloa, 1989). 
CUADRO 1. Localización de centros de trabajo de los jornaleros mixtecos en el noroeste de México y tipos de cultivos.

\begin{tabular}{lll}
\hline Estado & Localidad & Cultivo \\
\hline \multirow{2}{*}{ Baja California } & Valle de Mexicali & Algodón \\
& San Quintín & Tomate y hortalizas \\
Baja California Sur & Ciudad Constitución & Tomate y almeja \\
& Valle Sto. Domingo & Algodón \\
& Bahía de Vizcaíno & Tomate \\
& La Paz & Chile \\
Sonora & Obregón & Algodón \\
& Costa de Hermosillo & Uva \\
& Valle del Yaqui & Uva \\
Sinaloa & Valle de Culiacán & Tomate y hortalizas \\
& Guasave & $"$ \\
& Guamúchil & " \\
Nayarit & Valle La Virocha & Tomate y hortalizas \\
\hline
\end{tabular}

FUENTE: Elaborado por el autor.

en Guadalajara, Culiacán, costa de Hermosillo, Nogales, Tijuana, Ensenada, San Quintín y La Paz; asentamientos que han servido de base para apoyar su estrategia migratoria, creando paralelamente rutas que se han convertido en zonas de paso de cientos de jornaleros agrícolas. ${ }^{4}$ En Jalisco, Sinaloa, Sonora, Baja California y Baja California Sur, los residentes de origen mixteco suman poco más de 15,000 personas, $50 \%$ de los cuales radican en Tijuana. Los migrantes oaxaqueños han constituido una larga y extensa red migratoria que se extiende por toda la costa del Pacífico: desde Oaxaca y Guerrero hasta California y Washington (Kearney, 1986:71102; Chimal, et al., 1990: 33-45).

En el noroeste y en la frontera norte, los mixtecos se han integrado al mercado laboral urbano, que trasciende su tradicional experiencia ocupacional en la agricultura hacia los servicios y la industria. En el caso de los miembros

4 Una investigación realizada en Tijuana sobre estrategias familiares de reproducción social señala que el grupo étnico mixteco ha combinado la migración urbana con la rural, además de la ya conocida determinada por la selectividad por edad y sexo. Entre sus principales hipótesis, la autora sostiene que los cambios en la ruta migratoria también obedecen a criterios de organización familiar para la sobrevivencia de la unidad doméstica y del grupo, asociados a los cambios en el ciclo vital de hombres y mujeres (Velazco, 1989; 1990: 46-49). 
CUADRO 2. Localización de asentamientos de mixtecos en el Pacífico norte.

\begin{tabular}{|c|c|c|c|c|}
\hline \multicolumn{2}{|c|}{ Localización } & \multirow[b]{2}{*}{ Localidad } & \multicolumn{2}{|c|}{ Tipo de asentamiento } \\
\hline País & Estado & & Permanente & Temporal \\
\hline \multirow[t]{15}{*}{ México } & Jalisco & Guadalajara & $\mathrm{x}$ & \\
\hline & Nayarit & Valle La Virocha & & $\mathrm{x}$ \\
\hline & Sinaloa & Valle de Culiacán & $\mathrm{x}$ & $\mathrm{x}$ \\
\hline & & Guasave & & $\mathrm{x}$ \\
\hline & & Ruiz Cortines & & $\mathrm{x}$ \\
\hline & Sonora & Costa de Hermosillo & $x$ & $\mathrm{x}$ \\
\hline & & Nogales & $\mathrm{x}$ & \\
\hline & & Valle del Yaqui & & $\mathrm{x}$ \\
\hline & B.California & Valle de Mexicali & & $\mathrm{x}$ \\
\hline & & Valle de San Quintín & $\mathrm{x}$ & $\mathrm{x}$ \\
\hline & & Maneadero & $x$ & $\mathrm{x}$ \\
\hline & & Tijuana & $\mathrm{x}$ & \\
\hline & B.California S. & Valle de Vizcaíno & & $\mathrm{x}$ \\
\hline & & Valle de Santo Domingo & & $\mathrm{x}$ \\
\hline & & Ciudad Constitución & & $\mathrm{x}$ \\
\hline \multirow[t]{7}{*}{ E.U. } & California & San Diego & $\mathrm{x}$ & $\mathrm{x}$ \\
\hline & & Riverside & $\mathrm{x}$ & $\mathrm{x}$ \\
\hline & & Ventura & $\mathrm{x}$ & $\mathrm{x}$ \\
\hline & & Valle de San Joaquín & $x$ & $\mathrm{x}$ \\
\hline & & Fresno & $\mathrm{x}$ & $\mathrm{x}$ \\
\hline & & Arvin & $\mathrm{x}$ & $\mathrm{x}$ \\
\hline & & Livingstone & $\mathrm{x}$ & $\mathrm{x}$ \\
\hline
\end{tabular}

FUENTE: Elaborado por el autor.

jóvenes de las familias, especialmente de aquéllos que nacieron fuera de la Mixteca, los hombres se emplean como choferes, albañiles y jardineros, mientras que las mujeres recién se incorporan a la industria maquiladora.

En Culiacán, Sinaloa, un numeroso grupo de mixtecos se ha asentado en terrenos cercanos al valle agrícola, con el fin de emplearse en trabajos de carácter más o menos permanente, y en forma alternativa en las áreas rural y urbana (ver cuadro 2). En la costa de Hermosillo, Sonora, otro grupo de mixtecos levantaron un pequeño asentamiento en el poblado Miguel Alemán, con cerca de 1,000 residentes, con el propósito de conservar un trabajo más o menos estable en el cultivo de la uva. En Nogales, Sonora, en la colonia Oaxaca viven alrededor de 500 mixtecos; la mayoría de los hombres se emplean como peones en la industria de la construcción, mientras que las mujeres se dedican al comercio ambulante. 
En la historia y geografía de Baja California, su presencia es más notable. En Tijuana, los primeros asentamientos de mixtecos datan de los años setenta. En la actualidad, residen en seis colonias populares y trabajan principalmente como peones en la construcción; jardineros; trabajadoras domésticas y vendedores ambulantes. En la zona costa del estado, entre Ensenada y el Rosario, existen varios asentamientos permanentes, entre los que destacan Maneadero, Camalú, Vicente Guerrero, colonia Lázaro Cárdenas y San Quintín. Contrario a lo que sucede en Tijuana y asociado a la actividad económica de esas localidades casi la totalidad de los mixtecos son jornaleros agrícolas que laboran en cultivos hortícolas.

Aunque se sabe que pequeños grupos de mixtecos se emplean ocasionalmente en los estados de Oregon, Washington e incluso Alaska (en los Estados Unidos), su presencia laboral en California es más constante. Residen principalmente en ocho condados de este estado - San Diego, Riverside, Ventura, Tulare, Fresno, Madera, San Joaquín y Salinas-, en campamentos temporales y, en menor proporción, en asentamientos definitivos, desde donde se dirigen a los mercados laborales que demandan su presencia, principalmente en los campos agrícolas.

\section{MERCADOS LABORALES Y REDES SOCIALES}

De acuerdo con la localización de los mercados laborales, dentro y fuera del país, los trabajadores mixtecos conforman dos corrientes migratorias: una de migrantes internos y otra de migrantes internacionales. En términos de su inserción en el mercado laboral, una alta proporción de estos migrantes se emplean en campos agrícolas en el noroeste de México y en los Estados Unidos. Por otra parte, considerando los riesgos que implica cruzar la frontera sin documentos y las dificultades para que permanezcan mujeres y niños en el campamento y asentamientos temporales, la movilidad geográfica del grupo adquiere un carácter selectivo, por lo que, en su mayoría, quienes cruzan hacia el país vecino son los hombres jóvenes.

5 Es pertinente mencionar que diversos autores han mostrado que, en la actualidad, la migración laboral de trabajadores mexicanos hacia los Estados Unidos no proviene mayoritariamente ni de las comunidades rurales, ni de los sectores más pobres de México. En la historia de la emigración laboral de mexicanos hacia Estados Unidos, las tendencias de comportamiento y la composición interna de ese flujo internacional han variado. Jorge A. Bustamante señala al respecto: a) hasta 1969 el $80 \%$ de los migrantes indocumentados eran de origen rural; b) diez años después esta variable alcanzaba una proporción del $50 \%$; c) "los hallazgos más recientes indican que la proporción de migrantes de origen rural ha continuado decayendo". Los mixtecos forman parte de esa proporción decreciente de migrantes de origen rural que aún se dirigen a empleos agrícolas —en la última década cerca al 30 por ciento- y de una proporción menor de trabajadores con recursos limitados, 
El acceso a los empleos tradicionales en los mercados de trabajo urbano, comoel servicio doméstico y comercio ambulante para las mujeres y la industria de la construcción para los hombres, se ha ampliado a otros sectores de la economía formal e informal en las ciudades del noroeste y Pacífico norte. En territorio mexicano, las maquiladoras constituyen una nueva opción de trabajo para las mujeres. En los Estados Unidos, los mixtecos suplen a otros migrantes mexicanos y extranjeros que han dejado de emplearse en el campo en los cultivos de hortalizas y frutas frescas, pero también laboran en invernaderos cultivando plantas ornamentales y en pequeñas empresas que ofrecen servicios especializados de limpieza y mantenimiento.

El acceso al mercado laboral y las condiciones de la reproducción cotidiana, familiar y social, en uno y otro lado de la frontera implican para ellos problemas diferentes. Sin embargo, como grupo que se identifica por su cercanía étnica, conservan rasgos que han permitido su sobrevivencia colectiva. La continua movilidad de estos migrantes ha requerido de una extensa y bien integrada red de relaciones sociales. Las relaciones familiares y de comunidad disminuyen los costos del traslado, proporcionan una mayor seguridad al migrante y su familia, y ayudan a reproducir el proceso mismo de la migración. Estas redes sociales también implican la "extensión del pueblo" y sirven como punto de referencia fuera del lugar de origen.

Es característico que la llegada a los lugares de trabajo (en las ciudades y en los campos de cultivo) se realice en grupos familiares, procurando estar cerca de personas de sus mismos pueblos o rancherías. El concepto más amplio de familia y los lazos de amistad y parentesco, propios de la cultura mixteca y de otros grupos étnicos, cobran plena intensidad cuando de ello depende la sobrevivencia colectiva. Para un migrante, encontrar amigos o familiares de su mismo pueblo significa compartir satisfactores básicos de alimentación y alojamiento, además de compartir la experiencia migratoria a través de la transmisión de información sobre las posibilidades de éxito en el tránsito hacia el destino laboral. ${ }^{6}$

cuyas redes sociales apoyan su emigración hacia el vecino país. En años recientes, con la implementación de programas para contratación de trabajadores agrícolas y las modificaciones a la ley de inmigración de Estados Unidos (conocida como ley Simpson-Rodino), al igual que otros trabajadores mexicanos, los mixtecos obtuvieron permisos de trabajo y la posibilidad de obtener los derechos de inmigración para sus familiares (Véanse: Bustamante, 1986; Bustamente y Cornelius, 1989; García y Griego, 1988:8-9).

Un ejemplo de ello lo constituyen los asentamientos urbanos de mixtecos en la ciudad de Tijuana. Las familias residentes son una pieza clave en la red de relaciones sociales que permiten reproducir la migración, apoyando el alojamiento y atención de aquéllos que en su tránsito permanecen temporalmente en la ciudad. De manera inversa, para los mixtecos residentes en Tijuana, la posibilidad de enterarse de los acontecimientos que suceden en sus pueblos natales o al otro lado de la frontera constituye una información necesaria para la reproducción de los lazos que los unen a la comunidad. De esta forma, los migrantes transmiten información de ambos lados de la frontera que contribuye a reproducir su identidad como grupo. 
Sin embargo, estos mecanismos de apoyo mutuo y ayuda comunitaria frecuentemente son interpretados de manera equivocada en otros contextos. Un ejemplo ilustrativo lo constituye la acusación que se hace a los mixtecos como traficantes de indocumentados, cuando en defensa de su reproducción física y social tratan de apoyar a familiares y conocidos para cruzar la frontera y orientarlos para conseguir empleo.

\section{VIOLACIONES A SUS DERECHOS Y ACCIONES GUBERNAMEN- TALES}

La violación de los derechos humanos de los trabajadores migratorios es un hecho cotidiano. El migrante se hace más vulnerable al abuso de autoridad conforme se aleja de su comunidad de residencia habitual. La constitución mexicana le da el derecho de transitar libremente por su país como cualquier otro ciudadano; pero el abuso de autoridad, que culmina en la extorsión, coarta el ejercicio libre de ese derecho (Bustamante, 1991). Esta situación se hace más grave en el caso de los migrantes indígenas que sufren una mayor extorsión policial respecto a los no indígenas (Bustamante, 1990).

En su continua migratoria, la carencia de documentos de identificación facilita que los mixtecos sean acusados de indocumentados en su propio país. Una alta proporción de los migrantes no cuentan con documentos tales como acta de nacimiento, cartilla del servicio militar, credenciales del Instituto Mexicano del Seguro Social o de su centro de trabajo. Las cartas de presentación expedidas por los agentes municipales y comisarios ejidales carecen de validez oficial para las autoridades migratorias y policíacas. En el caso de los nacimientos de hijos ocurridos fuera de la Mixteca, con escasa frecuencia son notificados en las oficinas de registro civil.

Como migrantes y como trabajadores, los mixtecos sufren abusos de autoridades, de sus patrones y mayordomos, así como prácticas discriminatorias en ambos lados de la frontera. Sin embargo, gran parte de las violaciones a sus derechos ocurren en territorio nacional y son cometidas por las corporaciones policíacas. Los agentes policíacos interceptan a los migrantes en la vía pública, en las centrales de autobuses y estaciones de ferrocarril y, sobre todo, en las zonas cercanas a la línea internacional. La reciente desaparición de los retenes, activos en la "Campaña nacional contra el narcotráfico" de la Procuraduría General de la República, aligeraron la extorsión por parte de los elementos de la Policía Judicial Federal. A pesar de ello, aparecieron nuevas prácticas de extorsión a cargo de las brigadas móviles, ubicadas en centrales de autobuses y cruceros.

En el caso de los jornaleros mixtecos, el respeto a los derechos humanos y las faltas en el cumplimiento de la legislación laboral deben considerar 
que su condición de trabajadores migratorios se mezcla con su situación de indígenas. El 11 de diciembre de 1989, fue anunciado un programa del gobierno federal llamado "solidaridad para el regreso a México", mejor conocido como "programa paisano". Inició como un programa temporal dirigido a combatir abusos y extorsiones de que eran objeto los migrantes nacionales que laboraban, o residian, en los Estados Unidos y que retornaban a México para visitar a familiares con motivo de las fiestas decembrinas. A pesar de los aciertos de este programa, que a partir de enero de 1990 adquirió un carácter permanente, los migrantes indigenas continuaron expuestos a abusos cometidos por autoridades de migración, aduanas y de los distintos cuerpos policíacos. El abuso y la extorsión continuaron en Tijuana, peor aún en el caso de los mixtecos. Aquellos migrantes indigenas que cruzaban la frontera en autos o autobuses cran obligados a descender de los mismos para acreditar su nacionalidad y el valor de sus pertenencias, para ellos no había franquicia ni tampoco credibilidad al declararse ciudadanos mexicanos.

No son pocas ni recientes las ocasiones en que se han denunciado las condiciones infrahumanas en que viven los jornaleros agrícolas que laboran en los campos del noroeste del país; por el contrario, han sido escasas las acciones en materia de vivienda, salud, educación y seguridad social. En 1989, el gobierno del estado de Sinaloa puso en marcha el "Programa de desarrollo social para los jornaleros agrícolas de los valles de Sinaloa" (PRODESJA). El programa dio prioridad para resolver problemas de vivienda, salud, educación y asistencia social para los jomaleros y sus familias, en más de 100 campos agrícolas de la región. Con apoyo de FONHAPO, se construyeron cerca de 500 viviendas y se rehabilitaron un buen número de albergues. En el área de salud, en concertación con el Instituto Mexicano del Seguro Social, se instalaron consultorios médicos y unidades móviles de atención preventiva. Algunos servicios del sector educativo se reubicaron y otros se ampliaron. No obstante, aún existen rezagos en materia de impartición de justicia y, sobre todo, acciones muy limitadas para mejorar las condiciones de trabajo de mujeres y niños. ${ }^{7}$ En junio de 1990, y tomando como experiencia cl caso de Sinaloa, el

7 De acuerdo con el trabajo de apoyo y asesoría jurídica que realiza el foro de abogados egresados de la Universidad Autónoma de Baja California, en la zona costa de Baja California existen constantes irregularidades en la impartición de justicia y en la actuación de los cuerpos policíacos que afectan directamente a los migrantes. Entre las violaciones más frecuentes se encuentran detenciones y cateos sin orden judicial bajo pretexto de investigaciones relacionadas con narcotráfico, encarcelamiento indebido por delitos menores en la penitenciaría estatal, incomunicación y torturas físicas y morales realizadas por autoridades judiciales, firma de declaraciones y acusaciones sin la participación de abogado alguno y menos aún traductor bilingüe. (Ponencia presentada en la Audiencia Informativa sobre Derechos Humanos de los Trabajadores Migratorios Mexicanos, 1990). 
gobierno federal puso en marcha el "porgrama nacional de solidaridad" con los jornaleros agrícolas, programa que en 1991 tenía proyectado atender a los jornaleros de la costa de Hermosillo y el valle de San Quintín.

El 11 de agosto de 1990, los gobernadores de los estados de Oaxaca y Baja California firmaron un convenio de colaboración para atender las necesidades más apremiantes de los trabajadores agrícolas que laboran en la zona costa de Baja California. ${ }^{8}$ En el convenio se plantearon acciones de corto alcance que pretenden estimular un trabajo más amplio entre los dos gobiernos. Por lo pronto, los programas de solidaridad dirigidos a los jornaleros agrícolas del noroeste mexicano expresan buenas intenciones y plantean algunas acciones para abatir el amplio mapa que detalla el rezago social, que sin lugar a dudas refleja la enorme desigualdad entre agricultores y jornaleros, y una constante falta de atención hacia los trabajadores que han generado la riqueza agrícola de una de las regiones más productivas del país, considerada orgullo nacional por su alta tecnología y capacidad de exportación.

El principal argumento que justifica y demanda la intervención del Estado en la protección de los derechos humanos y laborales de los jornaleros agrícolas es la defensa del principio de igualdad jurídica y de impartición de justicia para todos los ciudadanos. La respuesta a los jornaleros agrícolas debe empezar en sus centros de trabajo y extenderse justamente a la defensa de sus derechos como mexicanos.

\section{ORGANIZACIONES PARA LA DEFENSA DE SUS DERECHOS}

En términos de organización social, los mixtecos han tenido que enfrentar y aprender las reglas de convivencia cotidiana propias de la cultura urbana, así como de las manifestaciones regionales de la cultura nacional y de la cultura norteamericana, y aquellas otras establecidas por las legislaciones mexicana y norteamericana. Este proceso de enfrentamiento y aprendizaje ha dado como resultado la formación de organizaciones civiles, laborales e incluso de acción y participación política para la defensa de sus derechos individuales, laborales y culturales. La migración de los mixtecos hacia el norte ha conformado una población residente y estacional, que no sólo ha incrementado su número, sino también su presencia social en ambos lados de la frontera.

8 En la reunión entre los gobernadores se consideró la urgente necesidad de diseñar una política que contemplara la atención al migrante agrícola y su familia en términos de los mínimos de bienestar. Algunas de las organizaciones sindicales involucradas propusieron la creación de una Comisión de Defensa de Derechos Humanos y Laborales, que hasta al momento no ha tenido un reconocimiento oficial. 
Tanto en la formación de sindicatos como de asociaciones civiles y organizaciones políticas, los mixtecos constituyen un grupo con una presencia notable en el noroeste del país y en California, en los Estados Unidos. Con cerca de una decena de organizaciones propias, los mixtecos extienden su acción desde el ámbito de la reproducción cotidiana de su cultura hasta el de la lucha política y sindical.

En defensa de sus raíces culturales, los mixtecos han conformado varias asociaciones con larga tradición en Baja California y California (E.U.A.). Las festividades del día de muertos en la colonia Obrera de Tijuana son una tradición que caracterizan el mozaico de las identidades que definen la de esta ciudad fronteriza. En Fresno, California, "Unidad Mixteca" y "Radio Bilingüe" son organizaciones pioneras que datan de 1950, cuyo objetivo ha sido preservar las tradiciones culturales de los mixtecos residentes en los Estados Unidos.

En su calidad de trabajadores agrícolas, los mixtecos se han integrado a las organizaciones sindicales tradicionales e independientes. En los valles agrícolas de Sinaloa y en el de San Quintín, el Sindicato Nacional Campesino, la Central Independiente de Obreros Agrícolas y Campesinos (CIOAC) y el Sindicato Nacional Independiente de Jornaleros y Obreros Agrícolas albergan en sus filas un gran número de jornaleros mixtecos. En Sonora y Baja California, además de integrarse a sindicatos, uniones y asociaciones laborales en la lucha de sus derechos como trabajadores del campo, los mixtecos han organizado asociaciones propias como trabajadores del sector servicios. Entre ellas destacan, la "Asociación Cívica Benito Juárez de Mixtecos Residentes en Tijuana" y la "Unión de vendedores ambulantes mixtecos de Nogales", ésta última perteneciente a la Central de Trabajadores de Sonora y a la Confederación Obrera Regional. En ambos casos se trata de vendedores dedicados al comercio ambulante, principalmente las artesanías.

En mayo de 1990, las asociaciones de trabajadores mixtecos de California (Estados Unidos de Norteamérica) realizaron su primer congreso en la ciudad de Los Ángeles, con objeto de formar un grupo único en la defensa de sus derechos laborales, civiles y culturales. Aunque existen diferencias entre ellos, comprensibles en el contexto de la diversidad de grupos dialectales y socioculturales que integran a los genéricamente llamados mixtecos, como trabajadores mexicanos en los Estados Unidos y como miembros de un grupo étnico con tradiciones fuertemente arraigadas y heredadas de sus comunidades de origen, han iniciado un nuevo diálogo para reforzar su organización. 


\section{CONSIDERACIONES FINALES}

La presencia de los mixtecos en el noroeste de México y en la costa del Pacífico de los Estados Unidos, a miles de kilómetros de su lugar de origen, es indicativo de varios procesos. En primer término, la emigración masiva de comunidades enteras y la migración como norma de vida cotidiana refleja la pobreza en que se encuentran las comunidades indígenas de Oaxaca y del país en general. En segundo lugar, el movimiento de los mixtecos supone también el movimiento de su cultura, de su idioma, de sus lazos personales y familiares, de su sentimiento de pertenencia a una comunidad, que en conjunto se transforman en mecanismos que han permitido la reproducción social del grupo étnico. Los elementos culturales que definen al grupo se trasladan junto con él en su obligada migración, convirtiéndose en base fundamental de sobrevivencia, en puentes de integración entre los migrantes, vividos con mayor intensidad precisamente por estar fuera del espacio geográfico de la comunidad. La información acumulada y la posibilidad de utilizarla con éxito depende de la experiencia de la comunidad, que imprime al movimiento migratorio una característica colectiva y no de trabajadores aislados.

En la defensa de sus derechos culturales, civiles y laborales, los trabajadores mixtecos se integran a organizaciones de larga tradición sindical y forman otras propias o independientes. Sin embargo, como otras minorias étnicas en el país y fuera de éste, sufren constantemente violacioncs a sus garantias como individuos, como ciudadanos y como trabajadores. Como indigenas son aún más vulnerables a los abusos de las autoridades, y con mayor frecuencia, sufren actos discriminatorios en la impartición de justicia y en el respeto a sus derechos humanos.

En Baja California, la reciente formación de la primera Procuraduría de los Derechos Humanos y Protección Ciudadana que se crea en el país ${ }^{9}$ es un hecho de gran trascendencia. El establecimiento de la sede de la procuraduría en Tijuana constituye un reconocimiento a la gravedad de la violación de los derechos humanos en esta ciudad, por donde cruzan más del $50 \%$ de los trabajadores mexicanos no documentados que se dirigen hacia los Estados Unidos, y también caracterizada por una alta inmigración interna. Los mixtecos, entre otros trabajadores migrantes internos e internacionales, son precisamente los más vulnerables por sus carencias económicas y su condición étnica; situación que paradojicamente ha constituido la base de la preservación de su identidad y su organización, generando la ampliación

9 En siete estados de la República Mexicana funcionan el mismo número de comisiones de derechos humanos, $\sin$ facultades ejecutivas. 
de sólidas redes sociales que han mantenido unida a la comunidad en su traslado temporal y en la decisión de asentarse definitivamente en el noroeste y Pacífico norte de México y los Estados Unidos.

\section{BIBLIOGRAFÍA}

ANGUIANO, María Eugenia. 1986. "Los mixtecos en Baja California". México indlgena. Año 2, no. 13.

BESSERER, Federico. 1989. "Mixtecos errantes". México Indlgena, no. 1 , nueva época.

BUSTAMANTE, Jorge. 1986. "Migración indocumentada México-Estados Unidos. Tendencias recientes de un mercado internacional de mano de obra". Ponencia presentada en la IV Reunión de Universidades de México y Estados Unidos de América sobre estudios fronterizos. Santa Fe, Nuevo México, abril 16-19.

- 1990. "Migración y extorsión policial". Ponencia presentada en la Audiencia informativa sobre derechos humanos de los trabajadores migratorios mexicanos, Senado de la República. Tijuana, B. C. Noviembre.

- 1991. "La extorsión en la frontera norte". Ponencia presentada en el seminario permanente Perspectivas de la modernización y del cambio social. COLEF. Diciembre, Tijuana B.C.

BUSTAMANTE, Jorge y Wayne Cornelius (coords.). 1989. Flujos migratorios mexicanos hacia Estados Unidos. México. Fondo de Cultura Económica.

BUTTERWORTH, Douglas. 1975. Tilantongo: Comunidad mixteca en transición. México, Instituto Nacional Indigenista.

CHIMAL, Carlos y Eniac Martínez. 1990. "Movimiento perpetuo. Mixtecos en California". México Indlgena, no. 4, nueva época.

CORONA Vázquez, Rodolfo. 1988. "Movilidad geográfica: búsqueda de bienestar", Demos. Carta demográfica sobre México.

GARCÍA y Griego, Manuel. 1988. "Cifras pequeñas, retos grandes", Demos. Carta demográfica sobre México.

GOBIERNO DEL ESTADO DE OAXACA. 1987. Lluvia, Tequio y Alimentos. Programa único de pequeña irrigación para las mixtecas oaxaqueñas. Oaxaca.

GOBIERNO DEL ESTADO DE SINALOA. 1989. "Programa de desarrollo social para los jornaleros agrícolas en los valles de Sinaloa". (manuscrito inédito). Sinaloa. 
KEARNEY, Michael. 1986. "Integration of the Mixteca and the Western U.S.-Mexican Border Region via Migratory Wage Labor", Regional Impacts of U.S.-Mexican Relations. San Diego, Center for U.S.Mexican Studies, University of California. (Monograph series, 16).

LII LEGISLATURA DEL SENADO DE LA REPÚBLICA. 1983. Informe de la Comisión de Asuntos Indígenas. Mixteca oaxaqueña. Cuadernos del Senado, 28. México.

VELAZCO Ortiz, Laura. 1989. Migración femenina y sobrevivencia familiar: el caso de los mixtecos en Tijuana. Tijuana, COLEF-PIEM (reporte de investigación).

- 1990. "Los mixtecos. Una cultura migrante". México Indigena, no. 4, nueva época.

ZAVIN, Carol, et al. 1990. "Indigenous Oaxacan Migrants in California Agriculture: A New Cycle of Poverty”. Davis, California Institute for Rural Studies, (mimeo). 